\title{
The new opportunities for high time resolution clinical TOF PET
}

\author{
Maurizio Conti ${ }^{1} \cdot$ Bernard Bendriem $^{1}$
}

Received: 21 December 2018 / Accepted: 1 February 2019 / Published online: 26 February 2019

(c) The Author(s) 2019

\begin{abstract}
Time-of-flight (TOF) positron emission tomography (PET) traces its origins to the beginning of PET itself. A brief historical review will be followed by a discussion of the recent development in silicon photomultipliers (SiPM), which has generated a quality leap in TOF performance. The principles of TOF PET physics and a discussion of the technical and clinical advantages of TOF PET compared to conventional PET will be presented: the concept of time resolution and its effect on TOF gain in signal-to-noise ratio, together with other properties such as reconstruction robustness to inconsistent data, convergence, and convergence speed. Finally, new exciting challenging applications enabled by very high-performance TOF PET scanners in the field of low count imaging, such as ${ }^{90} \mathrm{Y}$ imaging and theranostics, will be discussed.
\end{abstract}

Keywords Time-of-flight PET $\cdot \mathrm{SiPM} \cdot$ Reconstruction $\cdot$ Signal-to-noise ratio

\section{Introduction}

\section{An historical background}

Time-of-flight (TOF) measurement was suggested as early as 1969 by Brownell et al. [5]. The first practical scanners using this technology were built in the early 80 s by three leading groups. They used $\mathrm{CsF}[2]$ as the scintillator. CEALETI built a TOF PET, TTV01, in Grenoble [18] and the system was evaluated and used clinically at CEA-Orsay for at least a decade $[3,49]$. The second system was built and used at Washington University in Saint Louis [55] and the third one at the University of Texas in Houston [68, 69]. Another fast scintillator, the $\mathrm{BaF}_{2}$, was studied [28] and used by both CEA-LETI in another TOF PET, TTV03, [35], which was also used clinically for about a decade, and a CTI PET system [22]. Budinger [6], Tomitani [57] and Lewellen et al. [29] also contributed significantly in understanding the benefits of TOF. At the system level, measurement between 470 and 750 ps were reached. However, while the light scintillation properties of $\mathrm{CsF}$ or $\mathrm{BaF}_{2}$ were favorable for TOF performance, both the scintillators had low density that prevented the possibility of high spatial resolution and sampling. Moreover, the ultraviolet emission of $\mathrm{BaF}_{2}$ made light

Maurizio Conti

maurizioconti@siemens.com

1 Siemens Medical Solutions, Knoxville, USA collection difficult and required the use of more expensive photomultipliers (PMT) with quartz windows.

The need for high spatial resolution led to the parallel development of nonTOF PET during the late 70s and early $80 \mathrm{~s}$. The scintillator used was bismuth germanate $\left(\mathrm{Bi}_{4} \mathrm{Ge}_{3} 0_{12}\right.$ or BGO) [64], which has a high detection efficiency, acceptable light output, and a light emission wavelength of around $480 \mathrm{~nm}$. BGO became the standard in positron emission tomography (PET). However, its long decay time (300 ns) and low light output (about 8000 photons $\mathrm{MeV}^{-1}$ ) made it unusable in TOF PET. With the success of BGO-based PET systems, which enabled higher spatial resolution and higher sensitivity (although lower count rate than $\mathrm{CsF}$ or $\mathrm{BaF}_{2}$ ), TOF PET development came to a halt.

The discovery of new scintillators in the 90 s led to a new phase of TOF PET research and development. Cerium-doped lutetium oxyorthosilicate or $\mathrm{LSO}\left(\mathrm{Lu}_{2} \mathrm{SiO}_{5}\right)$ [36] has a relatively high light yield (about 30,000 photons $\left.\mathrm{MeV}^{-1}\right)$, high effective $Z\left(Z_{\text {eff }} \approx 66\right)$, high density $\left(7.4 \mathrm{~g} \mathrm{~cm}^{-3}\right)$ and a short decay time (40 ns). These characteristics allowed for higher spatial resolution and a higher count rate and preserved high sensitivity. This, combined with TOF information, pushed LSO to become the standard PET detector for one of the major PET scanner manufacturers $[20,66]$. The short decay time was immediately used to reduce the coincidence window from the $12 \mathrm{~ns}$ typical of BGO scanners to $6 \mathrm{~ns}$, and it was later reduced to $4.5 \mathrm{~ns}$ with the development of faster electronics. The 
short time coincidence window, now defined by the desired dimension of the transverse field-of-view (FOV), reduced the random coincidences in the data.

The LSO characteristics could also be used for a new generation of TOF PET scanners. Time resolution down to 300 ps was reported with two single LSO crystals in coincidence, making the TOF PET a viable solution [37]. First attempts to perform TOF reconstruction on a commercial LSO PET scanner showed a measurable gain in signal-to-noise ratio (SNR) due to the TOF reconstruction [9], even with a poor $1.2 \mathrm{~ns}$ time resolution.

In parallel, the more recent discovery of cerium-doped lanthanum bromide $\left(\mathrm{LaBr}_{3}\right)$ [60] opened another avenue for TOF instrumentation. While lanthanum bromide showed lower stopping power than LSO, it had a shorter decay time (16 ns), excellent energy resolution (about 3\% at $662 \mathrm{keV}$ ), and twice as much light output, making it an attractive candidate for TOF PET [51]. The first $\mathrm{LaBr}_{3}$ TOF scanner was developed at the University of Pennsylvania [24]. A 7.5\% energy resolution allowed for a reduced scatter fraction and a $460 \mathrm{ps}$ time resolution was measured. Further improvements in the triggering technique brought the time resolution to $420 \mathrm{ps}$, and there is evidence that time resolution could be brought down to 315-330 ps [25].

A first commercial TOF PET scanner, the Gemini TF PET/CT, was introduced by Philips in 2006 [52], and uses a LYSO scintillator crystal. LYSO is a material with a structure very similar to LSO: a fraction of the lutetium atoms in the crystal are replaced with yttrium. LYSO properties are also very similar to those of LSO, the main difference being the lower density due to yttrium's lower weight. The time resolution of the Gemini TF is 585 ps if measured with a low activity source.

At about the same time, the idea of extending the axial field-of view was proposed as a very efficient way to detect more radiation emitted inside the human body, for a given injection dose, in 3D acquisition mode [26].

Shortly after that, both General Electric and Siemens commercialized TOF PET/CT systems based on lutetiumbased scintillators with similar TOF performances between 520 and 550 ps [4, 27].

The combination of extending the axial field of view (FOV) and improving TOF performance have contributed to further progress in PET performance. The former leads to more count rates of true event, the latter leads to better use of such counts and thus to the reconstruction of images with better SNR, as will be discussed in the part II of this paper.

Additional valuable sources of information on TOF PET history and research can be found in some recent review papers available in the literature $[11,13,30,38$, $39,54,59]$.

\section{The recent development of SiPM-based TOF PET}

While the TOF capabilities in commercial systems was facilitated by the transition from the BGO scintillator to the fast LSO scintillator, the next evolution in reaching even better TOF performances is currently facilitated by the transition of the photodetector from a photomultiplier tube (PMT) to a silicon photomultiplier (SiPM). SiPM can provide a signal equivalent to PMTs through a similar gain but with the advantage of better energy and time resolution, and a much more compact packaging, as shown in Fig. 1. A very detailed review of the advantage of SiPM and other photodetectors has been written by Lewellen [31].

In brief, SiPMs consist of an array of photosentitive cells, called single-photon avalanche photodiodes (SPAD), which are operated in Geiger mode. Each time a photon hits one of the cells a charge avalanche is produced, resulting in a discrete signal. Similar to a PMT, the signal resulting from all the cells determines the energy of the incident gamma ray on the scintillator crystal. The signal from all the cells can be processed in different ways. In a so-called analog SiPM, signals from each individual cell are first summed up then digitized to produce timing and energy information. In a so-called digital SiPM, the signal from each cell is first digitized and then the desired information is obtained through summation of the number of cells that produced a single count. A detailed description of both devices as well as respective performance information for individual detectors has been published by Gundacker [19].

Through the recent use of SiPM photodetectors, TOF performance was improved to $300-400$ ps. The first system was introduced commercially by Philips in 2014 and a TOF performance of 345 ps was reported [41]. This was later further improved to $310 \mathrm{ps}$ [43]. A second system was recently developed by General Electric, and a TOF performance of 375 ps was reported [21].
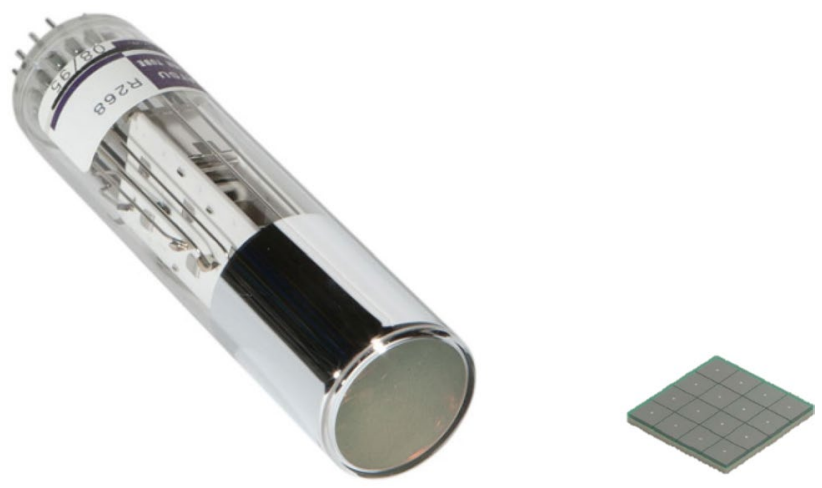

Fig. 1 Photomultiplier tube (PMT) (left) and silicon photomultiplier (SiPM) (right) 
While SiPM alone helped to improve TOF performance, other factors in the detector design also contribute to its overall performance. For example, the length of the scintillator crystal used, the type of coupling between the scintillator and the photodetector, as well the electronics processing the signal from the SiPMs all contribute to the detector performance. Siemens recently commercialized a new PET system using SiPM and a TOF performance of 210 ps was reported $[44,61]$. This new PET system also exploits the compactness of the SiPM using smaller $3.2 \mathrm{~mm}$ crystals, thus improving both TOF and spatial resolution at the same time.

In addition to TOF performance improvements, larger PET sensitivity through an extended axial FOV (to cover the whole body) is also being explored by other groups: it will open the path to new research opportunities, and likely create new clinical applications [8].

In the following section, we will expand the discussion on the concepts of TOF PET and the benefits of the recent technological advances.

\section{The principles and advantages of TOF PET}

\section{Principles of TOF PET}

Time-of-flight differences between two photons stopped in two detectors of the PET scanner are used to determine if the photons are in "time coincidence", and, therefore, associated with the annihilation of a positron-electron pair. If the detection time difference between two photons is smaller than a coincidence window (traditionally 4-10 ns), the two events are considered physically associated to the same annihilation event. A line-of-response (LOR) joining the two detectors is drawn, and the source of the positron is assumed to be located in a undetermined position along the line. Without more accurate TOF information, it is impossible to determine which voxel along the line is the source of the two photons; therefore, all the voxels along the line are given the same probability of emission. From the full set of LORs derived from the acquired data, analytical or iterative reconstruction algorithms are used to reconstruct the original activity distribution. Accurate measurement of the time difference is not needed and not available in conventional nonTOF PET.

On the contrary, TOF PET scanners are characterized by a more accurate measurement of the TOF difference, which allows better localization of the source of annihilation along the LOR. The precision is dominated by the time resolution of the TOF scanner or the uncertainty of the measurement of time difference, which ranges from 200 to $600 \mathrm{ps}$. In fact, the key performance parameter of a TOF PET scanner is the time resolution, typically measured as the full-width-half-maximum (FWHM) of the time distribution of a point source at the center of the FOV.

Since the TOF difference $t$ is proportional to the path length difference $x$ of the two photons, it could be used to know the "exact" position, along the LOR, of the positron annihilation (and if we ignore positron range, this is also the position of the radioisotope or tracer). How "exact" is obviously related to the time resolution $\Delta t$ of the scanner. Space and time uncertainty are proportional with $c$ and the speed of light: $\Delta x \approx c \Delta t / 2$.

If the time difference could be assessed with no uncertainty, no reconstruction algorithm would be necessary, since the position of the source along the line would be known. In today's TOF PET, such time measurement accuracy is not achievable, but the TOF information is still used, together with its estimated uncertainty. Each coincidence event is back projected along its LOR, using a bell-shaped probability curve like a Gaussian distribution, with mean position $x$ and FWHM $\Delta x$, derived from the measured time difference $t$ and time resolution $\Delta t$.

This TOF weighting process localizes the events and reduces the propagation of the statistical noise in the image. In each spatial position, only coincidence events with TOF differences consistent with such positions are accumulated. Noise events and randoms with inconsistent time information are filtered out. The overall effect is lower noise and higher contrast recovery; in other words, higher SNR. It is self-evident that the narrower the TOF kernel $\Delta t$ is, the more accurate the localization and the stronger the noise reduction.

In fact, the first studies on TOF PET demonstrated that TOF reduces the variance in the images of a factor proportional to $\Delta x / D$, where $D$ is the size of the object to be imaged $[6,57]$.

The ratio $\mathrm{Var}_{\text {nonTOF }} / \mathrm{Var}_{\mathrm{TOF}}$ is used as a figure of merit and called TOF gain. Budinger estimated such variance gain or TOF gain as [6]:

$\left(\mathrm{SNR}_{\text {gain }}\right)^{2}=\left(\frac{\operatorname{Var}_{\text {nonTOF }}}{\operatorname{Var}_{\mathrm{TOF}}}\right) \cong \frac{D}{c / 2^{\Delta t}}=\frac{D}{\Delta x}$.

Tomitani [57] mathematically derived the gain in image variance for a TOF confidence weighting filtered back projection (FBP) reconstruction, and obtained a gain reduced by a factor $\alpha^{2}=1 / 1.6$. More recently, Conti derived from experimental data a factor $\alpha^{2}=1 / 1.47$ [14]:

$\left(\mathrm{SNR}_{\text {gain }}\right)^{2}=\left(\frac{\operatorname{Var}_{\text {nonTOF }}}{\operatorname{Var}_{\mathrm{TOF}}}\right) \cong \alpha^{2} \frac{D}{c / 2^{\Delta t}}=\alpha^{2} \frac{D}{\Delta x}$.

Finally, an extension of the previous formulas has been introduced that shows a dependence of TOF gain with 
random fraction: higher gain is observed at higher random fractions [10, 17].

All these estimates are theoretically valid only for the variance measured at the center of a cylindrical source in the middle of the FOV that has a diameter $D$ and is uniform in attenuation properties and source distribution, and when an analytical or linear reconstruction is used, not an iterative method or a method in which image pixels are strongly correlated. Nevertheless, they represent a very good approximation of the TOF gain of a TOF PET scanner, and are commonly used also for general source distributions and iterative methods.

Since the SNR has been demonstrated to be proportional to the square root of the noise effective counts (NEC) in a PET scan [50], the TOF SNR gain is equivalent to a gain in counts, or $\mathrm{NEC}_{\text {gain }}=\mathrm{SNR}_{\text {gain }}^{2}$. In other terms, a TOF image is equivalent to a nonTOF image obtained with a larger number of counts. One can also consider TOF reconstruction as a "virtual counts or sensitivity amplifier" for a TOF PET scanner [13]. In describing the specifications of TOF PET scanners, sometimes the measured sensitivity and NEC performance are amplified by the estimated TOF gain, and defined "TOF-effective" or "effective" sensitivity and NEC.

To compare the image quality of a different time resolution, the same image quality phantom was imaged in the Siemens Biograph mCT PET/CT scanner with 527 ps intrinsic time resolution [27] and the new Siemens Biograph Vision with 210 ps intrinsic time resolution [44, $61]$. The phantom had six hot spheres $(5,8,10,13,17$, and $22 \mathrm{~mm}$ diameter) with a contrast of about $4: 1$, and a total activity in the background of about $50 \mathrm{MBq}$ of ${ }^{18} \mathrm{~F}$. The data were reconstructed TOF and nonTOF in both scanners. In Fig. 2, we show one transaxial slice of the image obtained with iterative reconstruction with resolution recovery and no post-reconstruction filter: a nonTOF image from the $\mathrm{mCT}$, a corresponding TOF image from the $\mathrm{mCT}$ (527 ps resolution), and a TOF image from the Biograph Vision (210 ps resolution). One can observe the improving image quality with improving time resolution: the smallest sphere is visible only using 210 ps time resolution TOF PET reconstruction.

\section{Robustness of TOF PET}

There is a whole series of possibilities opened by a characteristic of TOF PET that is intrinsically tied to the extra time information available. The recent literature has pointed out that TOF PET reconstruction is able to better handle data that are inconsistent, incomplete, or incorrect [13, 54]: TOF PET reconstruction is less sensitive to mismatched attenuation correction $[12,34,58]$, inaccurate normalization, and scatter correction $[12,63,65]$. To visually demonstrate this phenomenon, the same image quality phantom was scanned in the Siemens Biograph mCT PET/CT scanner (527 ps intrinsic time resolution) and the new Siemens Biograph Vision (210 ps intrinsic time resolution). The phantom had six hot spheres $(5,8,10,13,17$, and $22 \mathrm{~mm}$ diameter) with a contrast of about $4: 1$, and a total activity in the background of about $50 \mathrm{MBq}$ of ${ }^{18} \mathrm{~F}$. The data were reconstructed TOF and nonTOF in both scanners. To show the improving robustness of TOF reconstruction with improving time resolution, artifacts were intentionally introduced in the normalization, the attenuation correction, and scatter correction: we replaced the accurate corrections with inaccurate or artificially manipulated corrections. By doing so, artifacts appeared in the images. We observed that, while the inclusion of the TOF information dramatically decreases the artifacts compared to the corresponding nonTOF reconstruction, better time resolution produces images with even lower residual artifacts. Typical normalization ring artifacts are much reduced, attenuation artifacts almost disappear, and TOF images are less biased by inaccurate scatter correction.

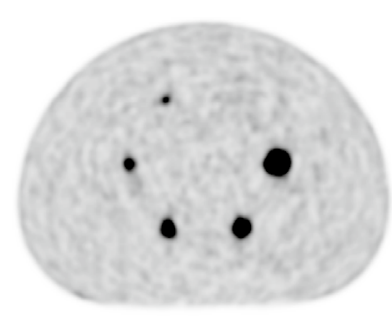

(a) nonTOF

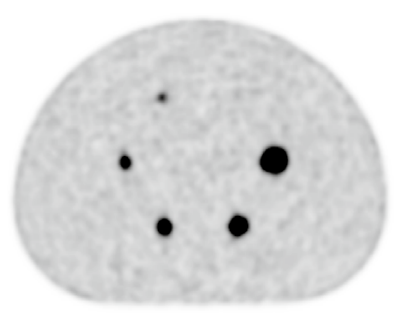

(b) TOF, 527ps

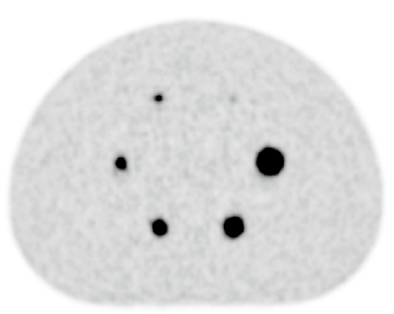

(c) TOF, 210ps
Fig. 2 Transaxial slices of an image quality phantom, reconstructed with high-resolution iterative reconstruction with resolution recovery. The scans were performed on a Siemens Biograph mCT PET/CT scanner (527 ps intrinsic time resolution) and the new Siemens Biograph Vision (210 ps intrinsic time resolution). From left to right: a non TOF image from mCT, $\mathbf{b}$ TOF image from Biograph mCT, and $\mathbf{c}$ TOF image from Biograph Vision. The phantom had six hot spheres $(5,8,10,13,17,22 \mathrm{~mm}$ diameter) with a contrast of about $4: 1$, and a total activity in the background of about $50 \mathrm{MBq}$ of ${ }^{18} \mathrm{~F}$ 


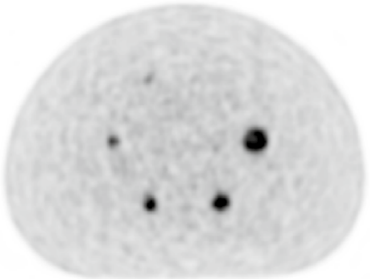

(a) nonTOF

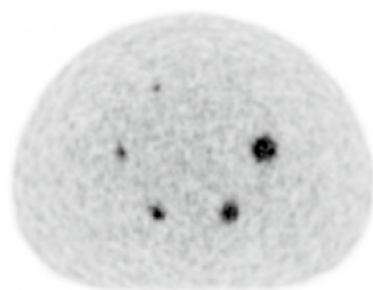

(c) nonTOF

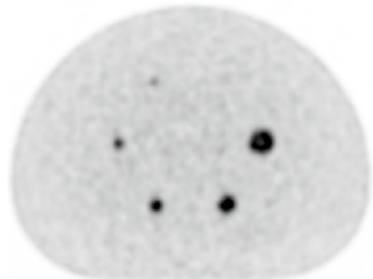

(b) TOF (527 ps)

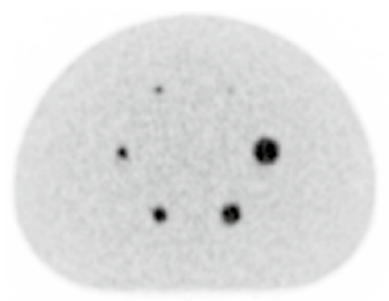

(d) TOF (210 ps)
Fig. 3 Transaxial slices of an image quality phantom, scanned on a Siemens Biograph mCT PET/CT scanner (527 ps intrinsic time resolution), and the new Siemens Biograph Vision (210 ps intrinsic time resolution). a Non TOF image from $\mathrm{mCT}$, b TOF image from $\mathrm{mCT}$, c nonTOF image from Biograph Vision, and $\mathbf{d}$ TOF image from Biograph Vision. The scatter correction was replaced by no scatter correction, which results in a typical bump of activity towards the center of the phantom

As an example, in Fig. 3, reconstruction was performed with no scatter correction, and one can observe that the typical hump in the center of the phantom is barely visible in the 210 ps TOF image.

Overall, TOF reconstruction provides a dramatic improvement in robustness of the image quality, since it is much less sensitive to any possible human or instrumentation error or even mismatch between PET and CT.

\section{Fast convergence of TOF PET}

It is well known that in iterative reconstruction, the choice of the number of iterations is somewhat arbitrary: both contrast recovery (or image sharpness) and noise increase with the iteration numbers, and typically a balance needs to be found.

The spatial information, provided by more precise TOF information of the new generation TOF PET scanners, results in a faster convergence of the TOF version of the same nonTOF iterative algorithm. One can take advantage of this property by pushing convergence higher and reaching higher contrast recovery at comparable noise levels (noiseequivalent images), or using a lower number of iterations for TOF reconstruction, with the result of having a much lower noise level for the same contrast recovery (contrastequivalent images), or any intermediate choice is possible.

The better the time resolution, the more dramatic and fast the convergence of TOF reconstruction is; see Fig. 4 [14].

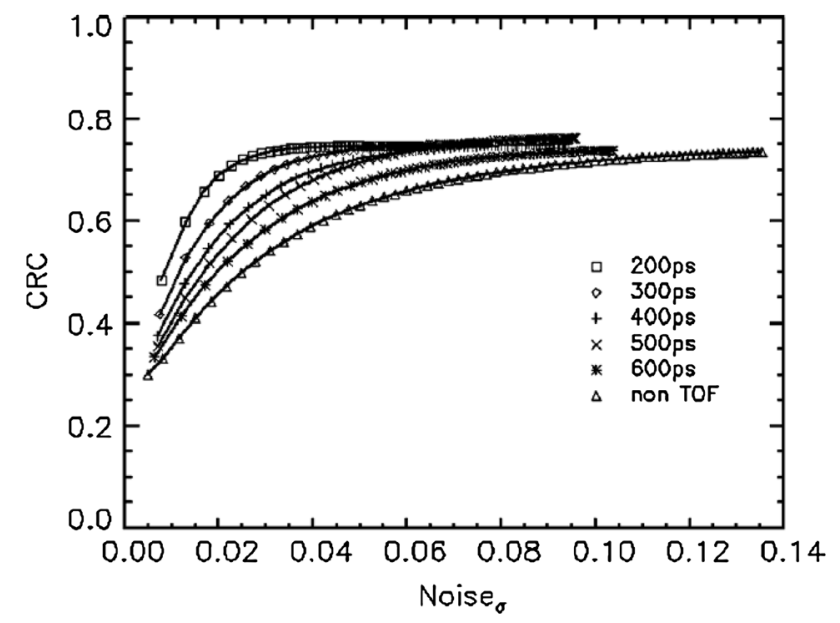

Fig. 4 Contrast recovery coefficient (CRC) vs. noise level, for a Monte Carlo simulation of a 10-mm sphere in a uniform cylinder (4:1 contrast). The nonTOF reconstruction and the TOF reconstructions: $600 \mathrm{ps}, 500 \mathrm{ps}, 400 \mathrm{ps}, 300 \mathrm{ps}$ and 200 ps. Iterative reconstruction: each point represents an iteration (from reference [14])

In this study, a uniform phantom with hot spheres $(4: 1)$ was simulated using Geant 4 . The contrast recovery of the smallest $10 \mathrm{~mm}$ sphere was studied vs. the iteration number or the noise in the background (image roughness).

\section{The clinical advantages of TOF PET}

\section{Clinical opportunities for TOF PET}

Advantages of the TOF-advanced performance include reduced variance or noise in the images, and higher quality images for clinical diagnosis. This translates also in better quantitative accuracy of lesion uptake and improves confidence in the clinical diagnosis [16]. Furthermore, it improves detectability of small lesions, as demonstrated in past phantom and patient studies [13, 23, 34 and 53]. Since TOF gain increases with patient size, TOF reconstruction acts as a weight equalizer, obtaining consistent image quality over a patient population, regardless of size and weight [34, 56]. With the improvement of TOF performances, the use of higher matrix size (and consequently smaller voxel size) has been facilitated. It is well known [42] that higher spatial resolution requires improved SNR to be clinically useful. Thus, the gain offered by TOF can be useful to allow smaller voxel size, which in turn improves detectability of lesions in clinical images [33, 41].

A second avenue to exploit the improved TOF performance is the further reduction of the injected dose. There is a worldwide trend toward dose reduction, motivated by concerns for patients, population exposure in general, and for exposure of medical professionals [40, 45]. In addition, 
pediatric applications become more attractive if the dose can be dramatically reduced. A lower dose would make the use of repeated PET scans to monitor the response to therapy more feasible. This could also improve therapy efficacy and reduce therapies that are ineffective or not necessary.

A third opportunity is the reduction of the scan time: scan time can be shortened while keeping the same image quality with better clinical workflow and added comfort for the patient. A lower cost and larger use of PET can be a consequence of this trend.

Obviously, not all three objectives can be obtained at the same time; a trade-off must be reached based on the patient case and the hospital needs.

\section{Emerging clinical applications enabled by high-performance TOF PET}

The dramatic development of theranostics and immunoPET has taken place naturally in TOF PET technology, because of the powerful reduction in image noise. Many theranostics and immunoPET tracers use long half-life radioisotopes, often associated with spurious prompt gammas, and typically at very low doses [46]. The combination of these three characteristics produces low count images, high noise, and contamination by a prompt gamma background, which is hard to correct [15]. TOF reconstruction provides noise reduction, and at the same time tends to filter out coincidence data that are inconsistent with the space-time distribution of pure coincidence events [12].

An example can be found in ${ }^{124}$ I PET imaging for thyroid cancer, which is used in association with ${ }^{131} \mathrm{I}$ radiotherapy. ${ }^{124} \mathrm{I}$ is a radioisotope with a high prompt gamma fraction (about 1/3 of the coincidence events) and long half-life $(100 \mathrm{~h})$. It is administered in small doses and, moreover, patients cannot be scanned for longer than 10-20 min. This results in very noisy images, as visible in Fig. 5. The patient in the figure has metastatic thyroid cancer, was injected with $40 \mathrm{MBq}$ of ${ }^{124} \mathrm{I}$, and was scanned for 4 min per bed on day 5 after injection. Total counts in the sinograms were very low $\left(10-100 \times 10^{3}\right)$. TOF reconstruction (Biograph mCT, $527 \mathrm{ps}$ intrinsic time resolution) improves quantification accuracy and reduces noise in the image, in particular the number of noise artifact foci in the chest and the abdomen are much reduced.

Another example of TOF PET technology as enabler of new clinical applications is the imaging of ${ }^{90} \mathrm{Y}$ radioembolization. Selective internal radionuclide therapy (SIRT) is a treatment for liver cancer based on injection of microspheres embedded with ${ }^{90} \mathrm{Y}$ therapeutic radioisotopes. ${ }^{90} \mathrm{Y}$ is characterized by a very small fraction of positron-electron pair annihilations (32 ppm), and, therefore, an extremely low PET signal (between $50 \times 10^{3}$ and $500 \times 10^{3}$ true counts per scan, which is orders of magnitude lower than a typical oncology scan). As soon as TOF PET became widely available in clinical practice, it showed its characteristics of superior quantitative performance [32], and quickly took off as the preferred technology. Multisite studies showed the superior capability of TOF PET scanners compared to older nonTOF models [7, 67]. Figure 6 shows an example of PET images of a patient undergoing SIRT treatment: noise reduction and better image quality characterize the TOF image compared to the nonTOF PET, in particular the extra hepatic artifact hot spots in the background tissue are greatly reduced.

PET/CT screening of patients at risk for lung cancer is a novel clinical application that might be enabled by highperformance TOF PET scanners. While CT lung cancer screening has been proposed [1], CT screening produces a false-positive rate of around 96\% [1]. The addition of metabolic information from PET could improve accuracy
Fig. 5 Coronal slice of PET image of a patient injected with $40 \mathrm{MBq}$ of ${ }^{124} \mathrm{I}$, day 5. Patient with differentiated thyroid cancer at high risk, bearing distant metastases. a NonTOF image and b TOF image. Data kindly provided by Walter Jentzen (University Clinic of Essen, Department of Nuclear Medicine, Germany). To better appreciate the difference of background noise, the color scale has been thresholded to $0.5 \%$ of the maximum value

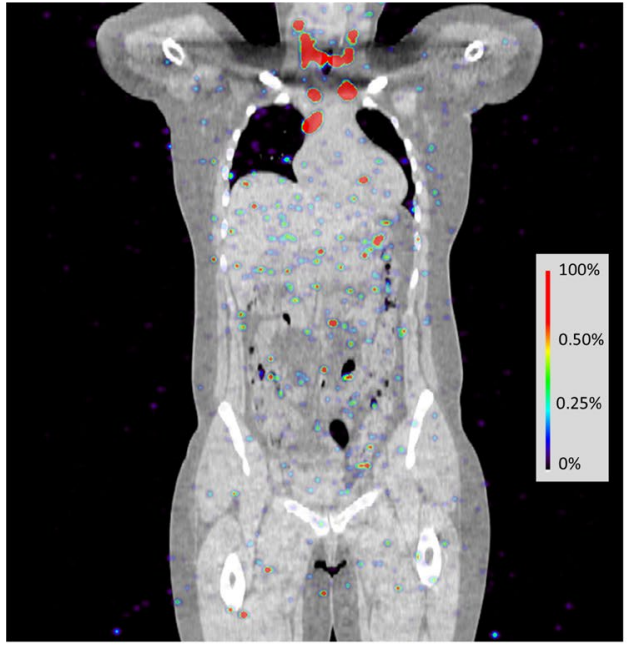

(a) non $\mathrm{TOF}$

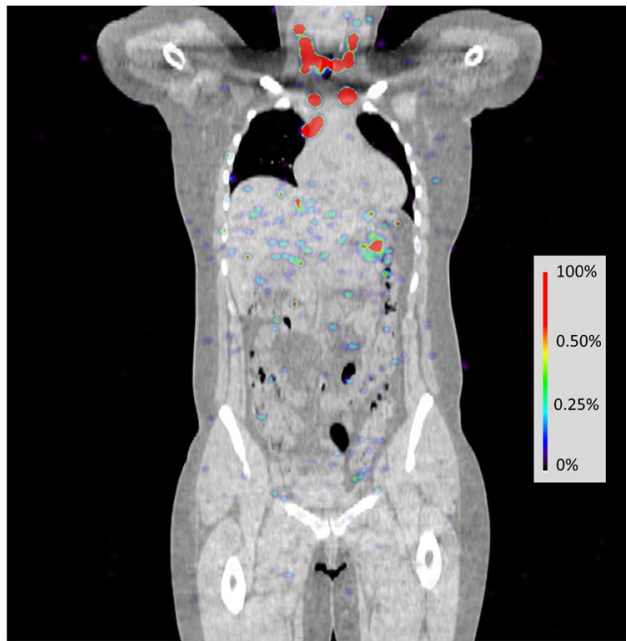

(b) TOF (527 ps) 
Fig. 6 Coronal slice of PET image of a patient liver undergoing SIRT treatment with

${ }^{90}$ Y-embedded resin microspheres in the liver. a NonTOF image and b TOF image. Data kindly provided by Kathy Willowson (Institute of Medical Physics, School of Physics, University of Sydney, Sydney, Australia). To better appreciate the difference of background noise, the color scale has been thresholded to $3 \%$ of the maximum value

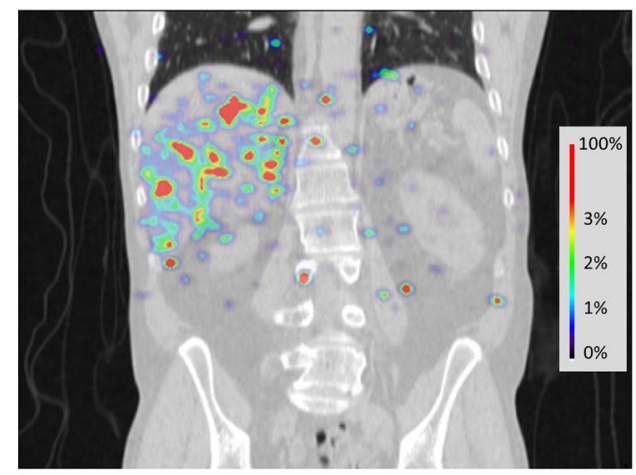

(a) nonTOF

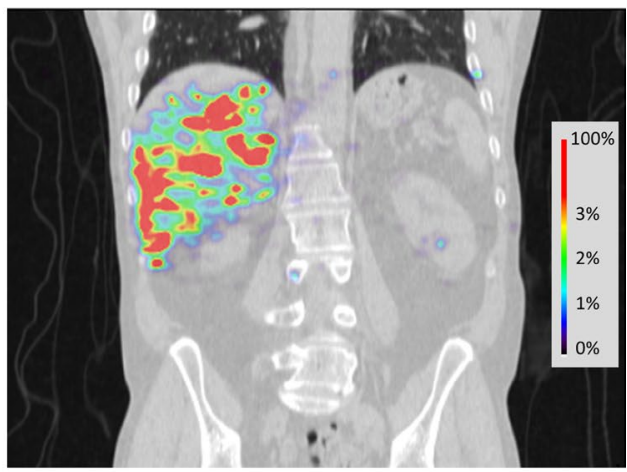

(b) TOF (527 ps) and greatly reduce false positives [48, 62]. This becomes feasible if the PET dose can be greatly reduced, to reduce the dose to the population of patients at risk. The new generations of PET/CT scanners, with about 200 ps time resolution, can provide a sensitivity gain (compared to conventional non TOF) of about 13 for a patient of about $40 \mathrm{~cm}$ abdominal size, which directly translates to an equivalent dose reduction. Dose reduction could be even larger, if a higher level of noise is acceptable for PET/CT screening [47]. PET/CT screening for populations at risk would, therefore, become feasible.

\section{Conclusions}

The introduction of lutetium-based scintillators and new photosensors, the SiPMs, has allowed a performance leap in time resolution. This translates into a considerable increase in TOF gain in SNR, unseen reconstruction robustness to inconsistent data, and increased convergence speed. New applications are enabled for PET scanners in the field of low count imaging, such as ${ }^{90} \mathrm{Y}$ imaging and theranostics, pediatric imaging, screening of patients at risk, and possibly many others.

We believe that even if the advantages of TOF PET technology have not yet been extensively studied and understood, the full adoption in clinical practice will continue to grow. While many scientific articles have already demonstrated the benefits of TOF, more clinical benefits and applications are yet to be exploited and advanced: this is an exciting time for PET scientists and clinicians.

Acknowledgements We would like to thank Walter Jentzen (University Clinic of Essen, Department of Nuclear Medicine, Germany), Kathy Willowson (Institute of Medical Physics, School of Physics, University of Sydney, Sydney, Australia), and Deepak Bharkhada (Siemens) for the patient and phantom images.

\section{Compliance with ethical standards}

Conflict of interest The authors are employees of Siemens Medical Solutions USA.

Ethical approval This article does not contain any studies with human participants or animals performed by any of the authors.

OpenAccess This article is distributed under the terms of the Creative Commons Attribution 4.0 International License (http://creativeco mmons.org/licenses/by/4.0/), which permits unrestricted use, distribution, and reproduction in any medium, provided you give appropriate credit to the original author(s) and the source, provide a link to the Creative Commons license, and indicate if changes were made.

\section{References}

1. Aberle DR, Adams AM, Berg CD et al (2011) Reduced lungcancer mortality with low-dose computed tomographic screening. N Engl J Med 365:395-409

2. Allemand R, Gresset C, Vacher J (1980) Potential advantages of a cesium fluoride scintillator for a time of flight positron camera. J Nucl Med 21:153-155

3. Bendriem B, Soussaline F, Campagnolo R, Verrey B, Wajnberg P, Syrota A (1986) A technique for the correction of scattered radiation in a PET system using time-of-flight information. J Comput Assist Tomogr 10(2):287-295

4. Bettinardi V, Presotto L et al (2011) Physical performance of the new hybrid PET/CT discovery-690. Med Phys 38(10):5394-5411

5. Brownell GL, Burnham CA et al (1969) New developments in positron scintigraphy and the application of cyclotron produced positron emitters. In: Proceedings of a symposium medical radioisotope scintigraphy, Salzburg Aug 1968, vol 1, pp 163-176

6. Budinger TF (1983) Time-of-flight positron emission tomography: status relative to conventional PET. J Nucl Med 24:73-78

7. Carlier T, Willowson K, Fourkal E, Bailey D, Doss M, Conti M (2015) ${ }^{90}$ Y PET imaging: exploring limitations and accuracy under conditions of low counts and high random fraction. Med Phys 42(7):4295-4309

8. Cherry S, Jones T, Karp JS, Qi J, Moses WW, Badawi R (2018) Total body PET: maximizing sensitivity to create new 
opportunities for clinical research and patient care. J Nucl Med 59:3-12

9. Conti M, Bendriem B, Casey M, Chen M, Kehren F, Michel C, Panin V (2005) First experimental results of time-of-flight reconstruction on an LSO PET scanner. Phys Med Biol 50:4507-4526

10. Conti M (2006) Effect of random reduction on signal-to-noiseratio in TOF PET. IEEE Trans Nucl Sci 53(3):1188-1193

11. Conti M (2009) State of the art and challenges of time-of-flight PET. Phys Med 25:1-11

12. Conti M (2011) Why is TOF PET reconstruction a more robust method in the presence of inconsistent data? Phys Med Biol $56: 155-168$

13. Conti M (2011) Focus on time-of-flight PET: the benefits of improved time resolution. Eur J Nucl Med Mol Imaging 38(6):1147-1157

14. Conti M, Westerwoudt V, Eriksson L (2013) Estimating image quality for future generations of TOF PET scanners. IEEE Trans Nucl Sci 60(1):87-94

15. Conti M, Eriksson L (2016) Physics of pure and non-pure positron emitters for PET; a review and a discussion. Eur J Nucl Med Mol Imaging Phys 3(1):1-17

16. Daube-Witherspoon ME, Surti S, Perkins AE, Karp JS (2014) Determination of accuracy and precision of lesion uptake measurements in human subjects with time-of-flight PET. J Nucl Med 55:602-607

17. Eriksson L, Conti M (2015) Randoms and TOF gain revisited. Phys Med Biol 60:1613-1623

18. Gariod R, Allemand R, Carmoreche E et al (1982) The LETI positron tomograph architecture and time of flight improvements. In: Proceeding of the workshop on time-of-flight tomography, Washington University: IEEE Publication, pp 25-29

19. Gundacker S, Auffray E, Jarron P, Meyer T, Lecoq P (2015) On the comparison of analog and digital SiPM readout in terms of expected timing performance. Nucl Instrum Methods Phys Res A 787:6-11

20. Herzog H, Tellmann L, Hocke C, Pietrzyk U, Casey ME, Kuwert T (2004) NEMA NU2-2001 guided performance evaluation of four Siemens ECAT PET scanners. IEEE Trans Nucl Sci 51(5):2662-2669

21. Hsu DF, Ilan E, Peterson WT et al (2017) Studies of a next-generation silicon-photomultiplier-based time-of-flight PET/CT system. J Nucl Med 58:1511-1518

22. Ishii K, Orihara H, Matsuzawa T, Binkley D, Nutt R (1990) High resolution time-of-flight positron emission tomograph. Rev Sci Instrum 61:3755

23. Kadrmas DJ, Casey ME, Conti M, Jakoby B, Lois C, Townsend DW (2009) Impact of time-of-flight on PET tumor detection. J Nucl Med 50(8):1315-1323

24. Karp JS, Kuhn A, Perkins AE, Surti S, Werner ME, Daube-Witherspoon ME, Popescu L, Vandenberghe S, Muehllehner G (2005) Characterization of a time-of-flight PET scanner based on lanthanum bromide. In: IEEE nuclear science symposium conference record, Puerto Rico, October 23-29

25. Kyba CCM, Wiener RI, Newcomer FM, Van Berg R, Dressnandt N, Karp JS (2007) Timing measurements from a TOF-PET scanner using local PMT triggering. In: 2007 nuclear science symposium conference record, Honolulu, Hawaii, October 27-November 3, 2007

26. Jakoby BW, Bercier Y, Watson CW, Bendriem B, Townsend DW (2009) Performance characteristics of a new LSO PET/CT scanner with extended axial field-of-view and PSF reconstruction. IEEE Trans Nucl Sci 56:633-639

27. Jakoby BW, Bercier Y, Conti M, Casey ME, Hayden C, Bendriem B, Townsend DW (2011) Physics performance and clinical investigation of the mCT time-of-flight PET/CT scanner. Phys Med Biol 56:2375-2389
28. Laval M, Moszynski M, Allemand R, Cormoreche E, Guinet P, Odru R, Vacher J (1983) Barium fluoride-inorganic scintillator for subnanosecond timing. Nucl Instrum Methods 206:169-176

29. Lewellen TK, Bice AN, Harrison RL, Pencke MD, Link JM (1988) Performance measurements of the SP3000/UW timeof-flight positron emission tomograph. IEEE Trans Nucl Sci 35(1):665-669

30. Lewellen TK (1998) Time-of-flight PET. Semin Nucl Med 28(3):268-275

31. Lewellen TK (2008) Recent development in PET detector technology. Phys Med Biol 53:R287-R2317

32. Lhommel R, van Elmbt L, Goffette P, Van den Eynde M, Jamar F, Pauwels S, Walrand S (2010) Feasibility of ${ }^{90} \mathrm{Y}$ TOF PET-based dosimetry in liver metastasis therapy using SIR-spheres. Eur J Nucl Med Mol Imaging 37:1654-1662

33. Li CY, Klohr S, Sadick H, Weiss C, Hoermann K, Schoenberg SO, Sadick M (2014) Effect of time-of-flight technique on the diagnostic performance of ${ }^{18} \mathrm{~F}$-FDG PET/CT for assessment of lymph node metastases in head and neck squamous cell carcinoma. J Nucl Med Technol 42:181-187

34. Lois C, Jakoby BW, Long MJ, Hubner KF, Barker DW, Casey ME, Conti M, Panin VY, Kadrmas DJ, Townsend DW (2010) An assessment of the impact of incorporating time-of-flight (TOF) information into clinical PET/CT imaging. J Nucl Med $51: 237-245$

35. Mazoyer B, Trebossen R, Shoukroun C et al (1990) Physical characteristics of TTV03, a new high spatial resolution time-of-flight positron tomograph. IEEE Trans Nucl Sci 37:778-782

36. Melcher CL, Schweitzer JS (1992) Cerium-doped lutetium orthosilicate: a fast, efficient new scintillator. IEEE Trans Nucl Sci 39:502-505

37. Moses WW, Derenzo SE (1999) Prospects for time-of-flight PET using LSO scintillator. IEEE Trans Nucl Sci 46(3):783-788

38. Moses WW (2003) Time of flight in PET revisited. IEEE Trans Nucl Sci 50(5):1325-1330

39. Muehllehner G, Karp JS (2006) Positron emission tomography. Phys Med Biol 51:R117-R137

40. Murray I, Kalemis A, Glennon J, Hasan S, Quraishi S, Beyer T et al (2010) Time-of-flight PET/CT using low-activity protocols: potential implications for cancer therapy monitoring. Eur J Nucl Med Mol Imaging 37:1643-1653

41. Nguyen NC, Vercher-Conejero JL, Sattar A et al (2015) Image quality and diagnostic performance of a digital PET prototype in patients with oncologic diseases: initial experience and comparison with analog PET. J Nucl Med 56:1378-1385

42. Phelps ME, Huang SC, Hoffman EJ et al (1982) An analysis of signal amplification using small detector in positron emission tomography. J Comp Assist Tomogr 6(3):551-565

43. Rausch I, Ruiz A, Valverde-Pascual I, Cal-Gonzales J, Beyer T, Carrio I (2018) Performance evaluation of the Philips vereos PET/ CT system according to the NEMA NU2-2012 standard. J Nucl Med. https://doi.org/10.2967/jnumed.118.215541

44. Reddin JS, Scheuermann JS, Bharkhada D, Smith AM, Casey ME, Conti M, Karp JS (2018) Performance evaluation of the SiPM based Siemens Biograph vision PET/CT system. In: IEEE nuclear science symposium and medical imaging conference record, Sydney, Australia, November 12-17

45. Roberts F, Gunawardana DH, Pathmaraj K, Wallace A, Mi T, Berlangieri SU et al (2005) Radiation dose to PET technologists and strategies to lower occupational exposure. J Nucl Med Technol 33:44-47

46. Rösch Frank, Herzog Hans, Qaim Syed M (2017) The beginning and development of the theranostic approach in nuclear medicine, as exemplified by the radionuclide pair ${ }^{86} \mathrm{Y}$ and ${ }^{90} \mathrm{Y}$. Pharmaceuticals 10:56 
47. Schaefferkoetter JD, Yan J, Sjöholm T, Townsend DW, Conti M, Tam JKC, Soo RA, Tham I (2017) Quantitative accuracy and lesion detectability of low-dose ${ }^{18}$ F-FDG PET for lung cancer screening. J Nucl Med 58:399-405

48. Schrevens L, Lorent N, Dooms C, Vansteenkiste J (2004) The role of PET scan in diagnosis, staging, and management of non-small cell lung cancer. Oncologist 9:633-643

49. Soussaline S, Comar D, Allemand R, Campagnolo R, Laval M, Vacher J (1985) New developments in positron emission tomography instrumentation using time-of-flight information. In: Gretz $\mathrm{T}$ et al (eds) The metabolism of the human brain studied with positron emission tomography. Raven Press, New York, pp 1-11

50. Strother SC, Casey ME, Hoffman EJ (1990) Measuring PET scanner sensitivity: relating count rates to image signal-tonoise ratios using noise equivalent counts. IEEE Trans Nucl Sci 37(2):783-788

51. Surti S, Karp JS, Muehllehner G, Raby PS (2003) Investigation of lanthanum scintillators for 3-D PET. IEEE Trans Nucl Sci 50(3):348-354

52. Surti S, Kuhn A, Werner ME, Perkins AE, Kolthammer J, Karp JS (2007) Performance of Philips Gemini TF PET/CT scanner with special consideration for its time-of-flight imaging capabilities. J Nucl Med 48(3):471-480

53. Surti S, Scheuermann J, El Fakhri G et al (2011) Impact of TOF PET on whole-body oncologic studies: a human observer detection and localization study. J Nucl Med 52:712-719

54. Surti S (2015) Update on time-of-flight PET imaging. J Nucl Med 56:98-105

55. Ter-Pogossian MM, Ficke DC, Yamamoto M et al (1982) Super PETT I: a positron emission tomograph utilizing photon time-offlight information. Trans Med Imaging 1:179-186

56. Taniguchi T, Akamatsu G, Kasahara Y, Mitsumoto K, Baba S, Tsutsui Y, Himuro K, Mikasa S, Kidera D, Sasaki M (2014) Improvement in PET/CT image quality in overweight patients with PSF and TOF. Ann Nucl Med. https://doi.org/10.1007/s1214 9-014-0912-z

57. Tomitani $T$ (1981) Image reconstruction and noise evaluation in photon time-of-flight assisted positron emission tomography. IEEE Trans Nucl Sci 28:4582-4588

58. Turkington TG, Wilson JM (2009) Attenuation artifacts and timeof-flight PET. In: IEEE nuclear science symposium and conference record, pp 2997-2999

59. Vandenberghe S, Mikhaylova E, D'Hoe E, Mollet P, Karp JS (2016) Recent developments in time-of-flight PET. Eur J Nucl Med Mol Imaging Phy 3:3

60. van Loef E, Dorenbos P, van Eijk CWE, Kramer KW, Gudel HU (2005) High-energy-resolution scintillator: $\mathrm{Ce}^{3+}$ activated $\mathrm{LaBr}_{3}$. Appl Phys Lett 79:1573
61. van Sluis J, de Jong J, Schaar J, Noordzij W, van Snick P, Dierckx R, Borra R, Willemsen A, Boellaard R (2019) Performance characterization of the digital Biograph Vision PET/CT system. J Nucl Med. https://doi.org/10.2967/jnumed.118.215418

62. Veronesi G, Bellomi M, Veronesi U et al (2007) Role of positron emission tomography scanning in the management of lung nodules detected at baseline computed tomography screening. Ann Thorac Surg 84:959-965

63. Wang W, Hu Z, Gualtieri EE, Parma MJ, Walsh ES, Sebok D, Hsieh YL, Tung CH, Song X, Griesmer JJ, Kolthammer JA, Popescu LM, Werner M, Karp JS, Gagnon D (2006) Systematic and distributed time-of-flight list mode PET reconstruction. In: IEEE nuclear science symposium and medical imaging conference record, San Diego, California, October 29-November 4, 2006

64. Weber MJ, Monchamp RR (1973) Luminescence of $\mathrm{Bi}_{4} \mathrm{Ge}_{3} \mathrm{O}_{12}$ spectral and decay properties. J Appl Phys 44:5495-5499

65. Werner ME, Surti S, Karp JS (2006) Implementation and evaluation of a 3D PET single scatter simulation with TOF modeling. In: IEEE nuclear science symposium and conference record, pp $1768-1773$

66. Wienhard K, Schmand M, Casey ME, Baker K, Bao J, Eriksson L, Jones WF, Knoess C, Lenox M, Lercher M, Luk P, Michel C, Reed JH, Richerzhagen N, Treffert J, Vollmar S, Young JW, Heiss WD, Nutt R (2002) The ECAT HRRT: performance and first clinical application of the new high resolution research tomograph. IEEE Trans Nucl Sci 49(1):104-110

67. Willowson KP, Bailey DL, Tapner M, The QUEST Investigator Team (2015) A multi-centre comparison of quantitative ${ }^{90} \mathrm{Y}$ PET/ CT for dosimetric purposes after radioembolisation. Eur J Nucl Med Mol Imag 1:1. https://doi.org/10.1007/s00259-015-3059-9

68. Wong WH, Mullani NA, Philippe EA, Hartz RK, Gould KL (1983) Image improvement and design optimization of the timeof-flight PET. J Nucl Med 24:52-60

69. Wong WH, Mullani NA, Philippe EA, Hartz RK, Bristow D, Yerian K, Gaeta JM, Ketharnavaz N (1984) Performance characteristics of the University of Texas TOFPET-I PET camera. J Nucl Med 25:46-47

Publisher's Note Springer Nature remains neutral with regard to jurisdictional claims in published maps and institutional affiliations. 